\title{
Eine Pilotstudie zur Wirksamkeit von Imiquimod in der Behandlung von Mollusca contagiosa bei Kindern
}

\section{Gabriele Feller \\ Christiane Bayerl \\ S. Goerdt}

\author{
A Pilot Study to Evaluate the Effectiveness of Imiquimod 5\% in the Treatment \\ of Mollusca Contagiosa in Children
}

\section{Zusammenfassung}

Die Behandlung von Mollusken ist auch heute noch für Arzt und Patient unbefriedigend, obwohl eine Reihe von Therapieoptionen zur Verfügung stehen. Insbesondere bei Kleinkindern mit ausgedehntem Befall sind die meisten Therapiemodalitäten nur bedingt einsetzbar, langwierig oder schmerzhaft. Die topische Anwendung von Imiquimod ist hier viel versprechend, da es bereits erfolgreich bei anogenitalen Warzen eingesetzt wurde. Der Wirkstoff (1-(2-methylpropyl)-1H-imidazo(4,5-c)quinolin-4amin) gehört zu den so genannten „Immune response modifiers“ und verstärkt unter anderem die zellvermittelte Immunantwort. In einer Pilotstudie untersuchten wir 15 Kinder im Alter von 4-11 Jahren über einen Beobachtungszeitraum von 16 Wochen. Die Behandlung wurde mit Imiquimod 5\% (Aldara Creme ${ }^{\circledR}$ ) $3 \times$ pro Woche durchgeführt. Bei 9 Kindern (69\%) zeigte sich ein Therapieansprechen, hiervon kam es bei 2 Kindern (15\%) zu einer kompletten Abheilung und bei 7 Kindern (54\%) zu einer deutlichen Reduktion der Viruswarzen. Bei 4 Kindern (31\%) kam es unter der Therapie mit Aldara Creme ${ }^{\circledR}$ zu keinem Ansprechen oder einer weiteren Zunahme von Dellwarzen. Bei 3 Kindern (23\%) mit partieller Remission konnte die Anzahl der Dellwarzen so weit reduziert werden, dass eine chirurgische Entfernung in Vollnarkose vermieden werden konnte. Insgesamt wurde die Therapie gut toleriert. Häufigste Nebenwirkungen waren lokale Rötung (85\%), Juckreiz (75\%), Brennen (23\%) und Schmerzen (8\%) im behandelten Areal. Bei 4 Kindern (31\%) kam es aufgrund lokaler Nebenwirkungen zu vorzeitigem Studienabbruch. Systemische Nebenwirkungen wurden nicht beobachtet. Die Applikation von Imiquimod bei Kindern mit Mollusca contagiosa stellt, insbesondere bei ausgedehntem Befall, eine neue Behandlungsalternative dar. Die optimale Dosierung und Behandlungsdauer müssen in weiteren Studien evaluiert werden.

\section{Abstract}

There is a wide variety of therapies for the treatment of mollusca contagiosa. In particular, treatment in young or anxious children is difficult, painful, and often time consuming. We investigated whether imiquimod 5\% (1-(2-methylpropyl)-1H-imidazo(4,5-c) quinolin-4 amin), a topical immune response modifier that stimulates the production of interferon- $\alpha$ and other cytokines, is an effective treatment for mollusca contagiosa. In this clinical trial we followed 15 children with multiple mollusca contagiosa, aged 4-11 years, for 16 weeks. The treatment was administered 3 times per week. The overall response rate was $69 \%$ ( 9 children). Out of these 2 patients (15\%) showed a complete remission and 7 children (54\%) had partial response with a remarkable reduction of mollusca contagiosa. 4 children (31\%) showed stable or progressive disease. In three children (23\%) with partial remission the large number of mollusca could be considerably reduced; thus surgical treatment in anaesthesia could be avoided. In general, the treatment was well tolerated. Side effects included erythema (85\%), itching (75\%), burning sensations (23\%) and pain ( $8 \%$ ). 4 children ( $31 \%$ ) stopped the treatment because of local side-effects. No systemic side effects were observed. The results of this study suggest that imiquimod $5 \%$ is a useful new therapy for the treatment of mollusca contagiosa in children, especially in severe cases. The dosing schedule and length of treatment for mollusca contagiosa require further evaluation. 
Mollusca contagiosa entstehen durch Infektion der Epidermis mit dem Poxvirus mollusci. Klinisch findet man isolierte oder in Gruppen stehende, breitbasig aufsitzende, blassrote Papeln mit zentraler Eindellung. Die Therapie von Mollusca contagiosa bei Kindern ist oft langwierig und von häufigen Rezidiven gekennzeichnet. Imiquimod ist ein neuer topischer „Immune response modifier“, der durch Stimulation der zellulären Immunantwort im Gewebe indirekt antivirale und antitumorale Effekte zeigt [16]. Dabei kommt es zur Induktion von Interferon $\alpha$, Interleukinen (IL-1, IL-5, IL-6, IL-8, IL-10, IL-12), TNF- $\alpha$ (Tumor necrosis factor $\alpha$ ), IL-1RA (Interleukin 1 receptor antagonist), G-CSF (Granulocyte colony-stimulating factor), GM-CSF (Granulocytemacrophage/colony-stimulating factor), MIP- $1 \alpha$ und MIP- $1 \beta$ (Macrophage inflammatory protein $1 \alpha$ und $1 \beta$ ) und MCP-1 (Macrophage chemotactic protein) [24]. Der genaue Wirkmechanismus ist jedoch noch nicht bekannt.

Derzeit ist Imiquimod für die lokale Therapie von Feigwarzen (Condylomata acuminata) zugelassen. Hier hat sich gezeigt, dass eine Anwendung $3 \times$ pro Woche über 8 Stunden für insgesamt 6-8 Wochen zu guten klinischen Resultaten führt [27]. Das Ziel dieser Untersuchung war es, die lokale Wirksamkeit von Imiquimod 5\% (Aldara Creme ${ }^{\circledR}$ ) in der Therapie von Mollusca contagiosa bei Kindern zu prüfen und die Verträglichkeit zu beurteilen.

\section{Material und Methode}

An der Pilotstudie mit Imiquimod nahmen 15 Kinder (6 weiblich, 9 männlich) im Alter von 4-11 Jahren mit klinisch gesicherten Mollusca contagiosa teil. Die Eltern der Kinder sowie die Kinder selbst wurden über die Art, das Ziel und den Ablauf der Anwendungsbeobachtung aufgeklärt. Für die Durchführung der Pilotstudie lag das Einverständnis der Ethikkommission vor.

Getestet wurde die lokale Wirkung von Aldara Creme (Imiquimod 5\%, Fa. 3M Medica). Bei der ersten Vorstellung wurden Einund Ausschlusskriterien überprüft. Es erfolgte eine allgemeine Untersuchung der Haut sowie die Erhebung der Krankengeschichte. Mollusca contagiosa wurden aufgrund des typischen klinischen Erscheinungsbildes zentral gedellter Papeln diagnostiziert. Die Anzahl der Mollusca contagiosa wurde ermittelt und fotodokumentiert. Zur Behandlung wurde Imiquimod $5 \%$ auf die mit Mollusca contagiosa befallenen Hautareale $3 \times$ pro Woche (Mo, Mi, Fr oder Di, Do, Sa) abends aufgetragen und morgens nach 8 Stunden abgewaschen. Der Therapiezeitraum betrug 16 Wochen. Die Patienten wurden in Woche 0 , Woche 1, Woche 4 , Woche 8 und abschließend in Woche 16 untersucht. Hierbei wurde der Lokalbefund evaluiert und fotodokumentiert. Lokale und systemische Nebenwirkungen wurden von Kindern bzw. Eltern erfasst. Bei Therapieansprechen und Abheilen der Mollusca contagiosa konnte die Studie vorzeitig beendet werden.

Die erhobenen Daten wurden mittels Wilcoxon-Test für abhängige Stichproben auf Signifikanz geprüft.

\section{Ergebnisse}

Im Rahmen der Studie wurden 15 Kinder im Alter von 4 bis 11 Jahren mit multiplen Mollusca contagiosa, durchschnittlich 3-55 Dellwarzen pro Kind, für 16 Wochen behandelt. Die Hauptlokalisationen fanden sich am Stamm, gluteal und im Bereich der Kniekehlen. Mollusca contagiosa im Gesicht oder im Genitalbereich wurden nicht mit Aldara Creme behandelt.

Bei 69\% (9 von 13 Kindern) konnte durch die Applikation von Aldara Creme $3 \times$ pro Woche ein Therapieansprechen erzielt werden. Davon waren 2 Kinder (15\%) nach 12 Wochen bereits erscheinungsfrei, und bei 7 Kindern zeigte sich nach 16 Wochen eine deutliche Reduktion der Warzenanzahl. 4 Kinder (31\%) zeigten kein Ansprechen bzw. eine Progression unter Therapie. 3 Kinder (23\%) beendeten die Studie vorzeitig aufgrund von lokalen Nebenwirkungen wie Brennen oder Schmerzen.

2 Kinder (15\%) nahmen wegen mangelnder Compliance der Eltern am Follow-up nach Studienbeginn nicht mehr teil und werden als Drop-out gewertet.

Die mittlere Behandlungsdauer betrug 12 Wochen.

Bei 3 von den 7 Kindern mit partiellem Ansprechen konnte durch die topische Anwendung von Imiquimod 5\% Creme der Lokalbefund so weit reduziert werden, dass eine chirurgische Abtragung durch Kürettage in Vollnarkose nicht mehr nötig war (Tab.1).

\section{Lokale Nebenwirkungen}

Als häufigste lokale Reaktion trat bei $85 \%$ der Kinder eine Rötung auf. Weitere lokale Nebenwirkungen waren Juckreiz (75\%), Brennen $(23 \%)$ und Schmerzen (8\%) im behandelten Areal.

\section{Rötung}

Eine lokale Rötung konnte erstmals 4 Wochen nach Behandlung bei 6 von 13 Kindern (46\%) beobachtet werden. Nach 8 Wochen zeigte sich bei 11 von 13 Kindern ( $85 \%$ ), bei Studienende bei 3 von 4 (75\%) Kindern eine Rötung.

\section{Juckreiz}

Mit zunehmender Behandlungsdauer zeigte sich auch eine deutliche Zunahme der Juckreizbeschwerden. Nach der ersten Behandlungswoche zeigte sich nur bei 1 von 13 Kindern $(7,7 \%)$ Juckreiz, in der vierten Woche bei 4 von 13 Kindern (31\%), in der achten Woche bei 8 von 13 Kindern (62\%), und am Studienende gaben noch 3 von 4 Kindern (75\%) Juckreiz im behandelten Areal an.

\section{Brennen}

Nach der ersten Behandlungswoche kam es bei keinem der Kinder zu Brennen im behandelten Areal. Nach vier Wochen gaben 1 von 13 Kindern (7,7\%), nach acht Wochen 3 von 13 Kindern (23\%) ein brennendes Gefühl an. Bei Studienabschluss wurde dieses Symptom von keinem Kind (0\%) mehr angegeben. 
Tab. 1 Ergebnisse der Anwendungsbeobachtung von topischer Imiquimod Creme (5\%) bei Patienten mit Mollusca contagiosa

\begin{tabular}{|c|c|c|c|c|c|c|c|}
\hline Patienten & A (Jahre) & $G$ & $\begin{array}{l}\text { Anzahl der MC } \\
\text { vor Therapie }\end{array}$ & $\begin{array}{l}\text { Anzahl der MC } \\
\text { nach Therapie }\end{array}$ & $\begin{array}{l}\text { Therapiedauer } \\
\text { (Wochen) }\end{array}$ & $\begin{array}{l}\text { Reduktionsrate } \\
\text { in \%* }\end{array}$ & Ansprechen \\
\hline 1 & 4 & $\mathrm{M}$ & 31 & 5 & 16 & $83,87 \%$ & PR \\
\hline 2 & 4 & $M$ & 7 & 9 & 12 & $0 \%$ & PD \\
\hline 3 & 10 & $M$ & 24 & 46 & 12 & $0 \%$ & PD \\
\hline 4 & 5 & $M$ & 55 & 19 & 16 & $65,45 \%$ & PR \\
\hline 5 & 4 & W & 43 & 4 & 16 & $90,69 \%$ & $P R$ \\
\hline 6 & 6 & $\mathrm{M}$ & 6 & 11 & 8 & $0 \%$ & PD \\
\hline 7 & 7 & W & 6 & 8 & 8 & $0 \%$ & PD \\
\hline 8 & 6 & W & 45 & 32 & 8 & $28,89 \%$ & PR \\
\hline 9 & 7 & W & 14 & 2 & 16 & $85,71 \%$ & PR \\
\hline 10 & 8 & $\mathrm{M}$ & 42 & 0 & 12 & $100 \%$ & $C R$ \\
\hline 11 & 11 & $M$ & 7 & 0 & 12 & $100 \%$ & CR \\
\hline 12 & 4 & $M$ & 10 & 6 & 8 & $40 \%$ & PR \\
\hline 13 & 10 & W & 30 & Drop-out & Drop-out & Drop-out & - \\
\hline 14 & 5 & W & 3 & 1 & 8 & $66,67 \%$ & PR \\
\hline 15 & 7 & $M$ & 3 & Drop-out & Drop-out & Drop-out & - \\
\hline Median & 6 & & 14 & 6 & 12 & $65,45 \%$ & \\
\hline Mittelwert & 6,3 & & 18,7 & 11,8 & 10 & $44,71 \%$ & \\
\hline
\end{tabular}

* Reduktionsrate: Anzahl der MC vor Th./Anzahl der MC nach Th. × 100; MC: Mollusca contagiosa; CR: komplette Remission; PR: partiale Remission; PD: Progression; G: Geschlecht; A: Alter; M: männlich; W: weiblich

Tab. 2 Lokale Nebenwirkungen von Imiquimod 5\% in Abhängigkeit von der Behandlungsdauer (Applikation $3 \times /$ Woche) in Prozent

\begin{tabular}{|c|c|c|c|c|}
\hline \multicolumn{5}{|c|}{ Behandlungsdauer } \\
\hline Reaktionen in \% & Woche 1 & Woche 4 & Woche 8 & Woche 16 \\
\hline Juckreiz & $8(1 / 13)$ & $33(4 / 13)$ & $62(8 / 13)$ & $75(3 / 4)$ \\
\hline Brennen & $0(0 / 13)$ & $8(1 / 13)$ & $23(3 / 13)$ & $0(0 / 4)$ \\
\hline Rötung & $0(0 / 13)$ & $46(6 / 13)$ & $85(11 / 13)$ & $75(3 / 4)$ \\
\hline Schmerzen & $0(0 / 13)$ & $8(1 / 13)$ & $8(1 / 13)$ & $0(0 / 4)$ \\
\hline
\end{tabular}

Tab. 3 Weitere Einsatzmöglichkeiten von Imiquimod bei tumoralen, viralen und parasitären Hauterkrankungen

\begin{tabular}{|c|c|c|c|c|}
\hline Indikation & Art der Studie & Patientenanzahl & Ansprechrate & Literatur \\
\hline Basaliome & $\begin{array}{l}\text { offen, } \\
\text { multizentrisch, } \\
\text { randomisiert, } \\
\text { 4-armig, } \\
\text { dosisabhängig }\end{array}$ & $n=99$ & $\begin{array}{l}100 \% \text { CR }(\mathrm{l} .2 \times / \text { d) } \\
87,9 \% \text { CR }(\mathrm{l} .1 \times / \text { d) } \\
73,3 \% \text { CR }(\mathrm{l} .2 \times / \mathrm{d}-3 \times / \text { Wo }) \\
69,7 \% \text { CR }(\mathrm{l} .1 \times / \mathrm{d}-3 \times / \text { Wo })\end{array}$ & Marks R [18] \\
\hline M. Bowen & offen, Phase II & $n=16$ & $93 \%$ CR & Mackenzie-Wood A [20] \\
\hline aktinische Keratosen & Fallserie & $n=6$ & $100 \%$ CR nach $6-8$ Wo. Th. & Stockfleth E [26] \\
\hline $\begin{array}{l}\text { intraepitheliale Neoplasien } \\
\text { (Vulva, vaginal, Cervix uteri) }\end{array}$ & Fallserie & $n=8$ & $\begin{array}{l}75 \% \\
50 \% \text { CR } \\
25 \% \mathrm{PR}\end{array}$ & Diaz Arrastia C [8] \\
\hline Lentigo maligna & Fallbericht & $\mathrm{n}=1$ & $100 \%$ & Ahmed I [1] \\
\hline Verrucae vulgares & offen, prospektiv & $\mathrm{n}=50$ & $\begin{array}{l}56 \% \\
30 \% \text { CR } \\
26 \% \text { PR }\end{array}$ & Hengge UR [13] \\
\hline Verrucae planae & Fallbericht & $\mathrm{n}=1$ & $100 \%$ CR nach 6 Wochen Th. & Oster-Schmidt C [22] \\
\hline Herpes simplex & Fallbericht & $\mathrm{n}=1$ & $100 \%$ CR nach 1 Woche Th. & Gilbert J [11] \\
\hline kutane Leishmaniose & offen, prospektiv & $\mathrm{n}=12$ & $\begin{array}{l}100 \% \\
\text { nach } 6 \text { Mon. } 90 \% \text { CR }\end{array}$ & Arevalo I [2] \\
\hline
\end{tabular}




\section{Schmerzen}

Während der Studie kam es nur bei einem Kind in Woche 4 (7,7\%) und Woche 8 (7,7\%) zu Schmerzen im Behandlungsareal. Hierbei handelte es sich um eine lokale, stark erosive Entzündungsreaktion bei einem Mädchen mit Mollusca contagiosa im perigenitalen Hautbereich, das über heftige Schmerzen beim Wasserlassen klagte (Tab. 2).

\section{Systemische Nebenwirkungen}

Bei keinem der behandelten Kinder kam es während der Behandlung mit Aldara Creme ${ }^{\circledR}$ zum Auftreten von systemischen Nebenwirkungen wie grippeähnlichen Symptomen, Myalgien oder Kopfschmerzen.

\section{Diskussion}

Warzen, Condylome und Mollusken werden häufig bei immunsupprimierten Patienten beobachtet.

Insbesondere Kleinkinder und Kinder leiden an Mollusca contagiosa. Der Grund ist vermutlich eine unzureichende zelluläre Immunabwehr, besonders bei atopischer Disposition. Bei Erwachsenen treten Warzen, Condylome und Mollusken vermehrt bei chronischer Immunsuppression (HIV-Infektion, iatrogene Immunsuppression, z.B. durch Knochenmarkstransplantation oder Chemotherapie) auf.

Eine Reihe von immunstimulierenden Substanzen wie z.B. topische $\beta$-Interferone zeigten bereits bei HPV-induzierten Viren Erfolge [6]. Auch Imiquimod (Aldara ${ }^{\circledR}$ ) wurde effektiv bei anogenitalen Warzen mit Therapieansprechraten von $37-50 \%$ eingesetzt $[4,9]$. In der vorliegenden Pilotstudie konnten wir zeigen, dass es auch bei Mollusken zu einem deutlichen Ansprechen (69\%) kommt. Die Behandlung erfolgte wie bei Genitalwarzen durch eine dreimalige Applikation pro Woche über einen Zeitraum von 16 Wochen. Bei immunkompetenten Kindern im Alter von 4-11 Jahren konnte mit dieser Therapie eine komplette Remission von $15 \%$ erzielt werden. Bei weiteren $54 \%$ der Kinder kam es unter der Behandlung zu einer Reduktion der Warzenanzahl. Vergleichbare Studien zur Therapie von Mollusca contagiosa mit Imiquimod $5 \%$ wurden z. B. von Hengge et al. durchgeführt [13]. Dort fand sich jedoch eine höhere Rate für Patienten mit kompletter Remission (53\%) bei Mollusca contagiosa. Ebenfalls konnten Liota et al. bei nicht immunsupprimierten Kindern eine komplette Remission bei 6 von 13 Kindern (46\%) feststellen [17]. Bei den oben genannten Studien wurde Imiquimod durch Selbstapplikation angewendet. In der vorliegenden Studie wurde das Präparat jeweils von einem Elternteil aufgetragen. Vermutlich ist auch ein Anteil der Non-responder auf Non-Compliance zurückzuführen. Auch Nebenwirkungen wie Rötung, Juckreiz und Brennen können hier verantwortlich für eine Minderung der Compliance sein.

Unter der Behandlung der Mollusca contagiosa mit Imiquimod $5 \%$ kam es bei keinem der Kinder zu systemischen Nebenwirkungen wie z.B. grippeähnlichen Symptomen, Myalgien oder Kopfschmerzen. Diese Ergebnisse sind mit der in der Literatur angegebenen niedrigen systemischen Resorptionsrate von Imi- quimod zu vereinbaren. Sauder wies nach einer einmaligen Applikation von Imiquimod weniger als $1 \%$ des Wirkstoffes im Urin nach [24].

Als häufigste lokale Reaktion trat nach 8 Wochen eine Rötung im behandelten Areal bei $85 \%$ der Kinder auf. Je stärker die Entzündungsreaktion auftrat, desto schneller zeigten die befallenen Läsionen Abheilungstendenz. Areale mit nur mildem Erythem reagierten auf die Therapie entsprechend langsamer. Diese Beobachtungen stehen mit den Ergebnissen von Smith in Einklang, der bei Kindern mit starker Lokalreaktion auf Imiquimod ein schnelleres Abheilen der Dellwarzen beobachtete [25].

Bisherige Behandlungsoptionen umfassen bei Kindern hauptsächlich Kürettagen, Kryotherapie oder Verätzung mit Trichloressigsäure. In speziellen Zentren wird auch die Entfernung mittels gepulstem Farbstofflaser durchgeführt, allerdings ist diese Therapie mit vergleichsweise hohen Behandlungskosten verbunden und nur Einzelfällen vorbehalten [12]. Die lokale oder systemische Gabe von Cidofovir, einem azyklischen Nukleosidphosphonat, wird vor allem bei immunsupprimierten Patienten eingesetzt $[3,19,29]$. Der topische Einsatz von Cantharidin [14,15], Potassium Hydroxid 5\% [23] oder Sibernitratpaste [21] spielt in Deutschland eine untergeordnete Rolle. Systematische Studien zur Therapie von Mollusca contagiosa stehen bislang nicht zur Verfügung [13].

Im Vergleich mit den allgemein üblichen Behandlungen (Kürettagen, Kryotherapie), die alle mit Wundschmerzen oder Brennen einhergehen und in einigen Fällen sogar eine Vollnarkose erfordern, erscheint die Therapie mit Imiquimod weniger traumatisch und aus diesem Grunde für Kinder geeignet.

Besonders erfolgreich zeigte sich der Einsatz bei 3 Kindern, die aufgrund des ausgedehnten Befalles für eine Vollnarkose vorgesehen waren. Hier kam es unter der Therapie mit Imiquimod innerhalb von 16 Wochen zu einer deutlichen Reduktion der Dellwarzen. Alle 3 Kinder wendeten Imiquimod auch nach Studienende weiter $3 \times$ pro Woche an und waren nach 6 bzw. 8 Monaten erscheinungsfrei.

Imiquimod ist ein neues Medikament aus der Gruppe der „Immune response modifier“. Die Pharmakoaktivität resultiert hauptsächlich aus der Induktion von Interferon- $\alpha$ und anderen Zytokinen in der Haut, die unterschiedliche Immunantworten des humoralen Immunsystems beeinflussen. Darüber hinaus stimuliert Imiquimod auch die zelluläre Immunantwort, die besonders für die Kontrolle von viralen Infektionen und Tumoren eine wichtige Rolle spielt $[7,28]$.

Ausgehend von diesen Erkenntnissen wurde Imiquimod bei verschiedenen Viruserkrankungen der Haut sowie bei Hauttumoren untersucht [10].

Über ein Therapieansprechen wird einerseits in der Behandlung viraler Erkrankungen wie Herpes simplex [11], Verrucae planae [22] und Verrucae vulgares [13] berichtet. Andererseits konnte durch die Applikation von Imiquimod auch bei tumoralen Veränderungen wie Basaliomen [18], aktinischen Keratosen [26], Lentigo maligna [1], intraepithelialen Neoplasien [8] und M. Bowen [20] durch die immunmodulierende Eigenschaft ein positiver Ef- 


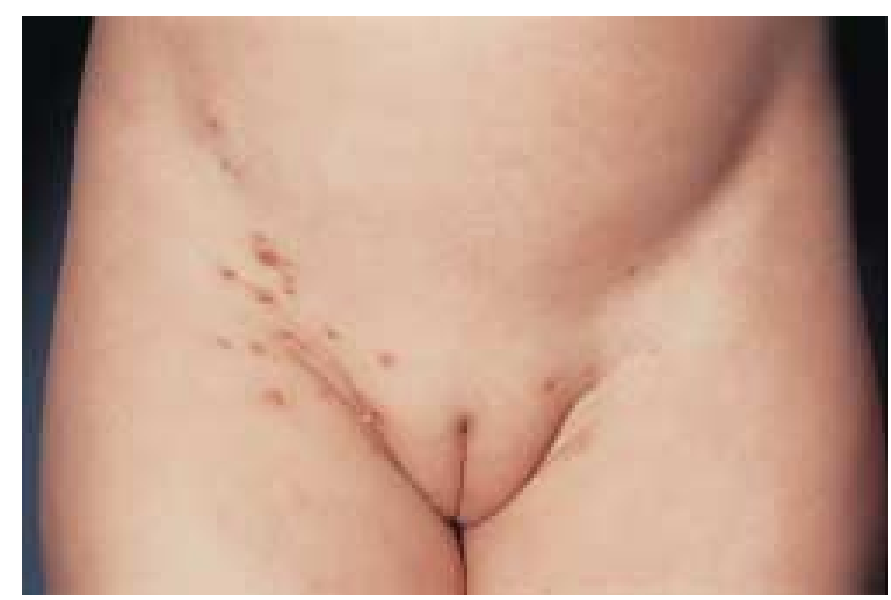

Abb. 1 4-jähriges Mädchen mit multiplen Mollusca contagiosa perigenital und perianal 4 Wochen nach Therapie mit Imiquimod 5\%.

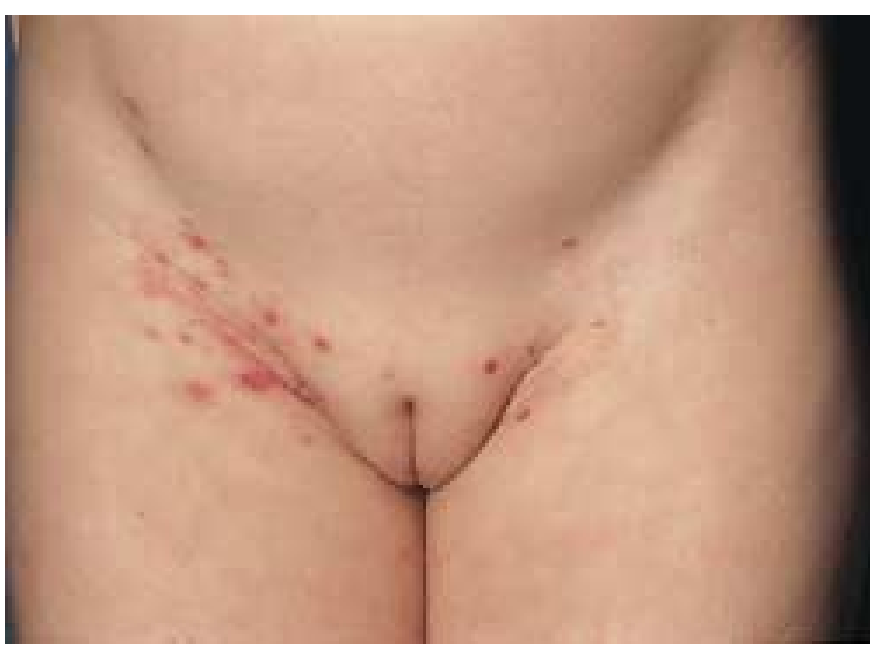

Abb. 28 Wochen nach Therapie mit Imiquimod 5\% mit deutlichem Erythem und Erosionen.

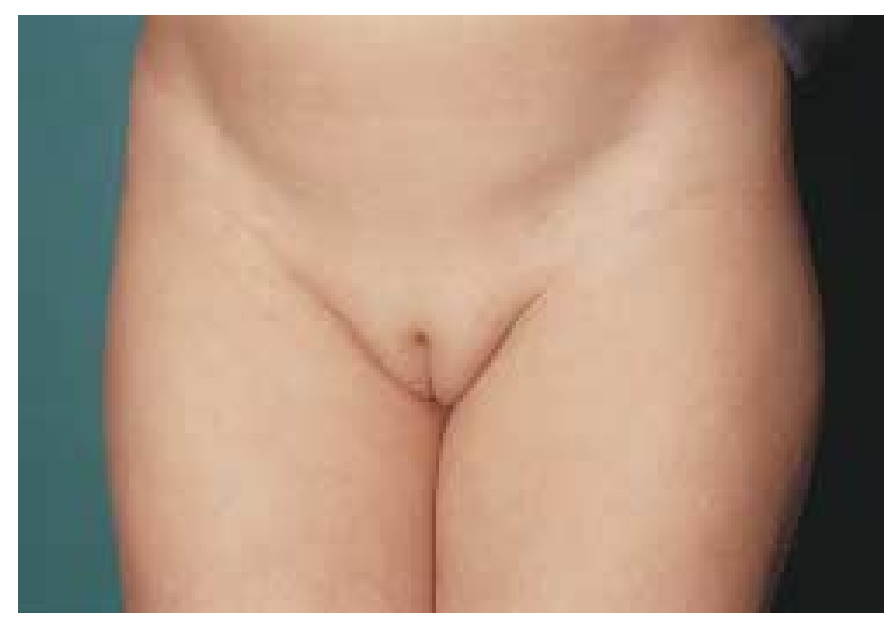

Abb. 3 Nach 16 Wochen Therapie mit Imiquimod 5\%, Therapiekontrolle 3 Monate später, alle Mollusca contagiosa sind abgeheilt.

fekt gezeigt werden. Auch bei parasitär bedingten Erkrankungen wie der kutanen Leishmaniose ist ein Therapieerfolg beschrieben [2]. Trotz der vielfältigen in Studien nachgewiesenen antiviralen und antitumoralen Eigenschaften ist der Wirkstoff Imiquimod

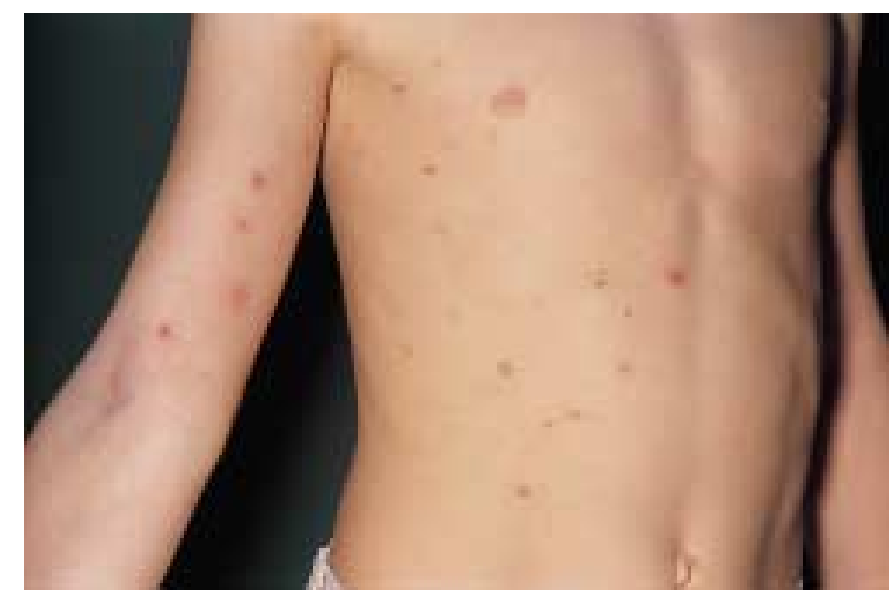

Abb. 4 8-jähriger Junge mit disseminierten Mollusca contagiosa am gesamten Integument vor Therapie mit Imiquimod 5\%.

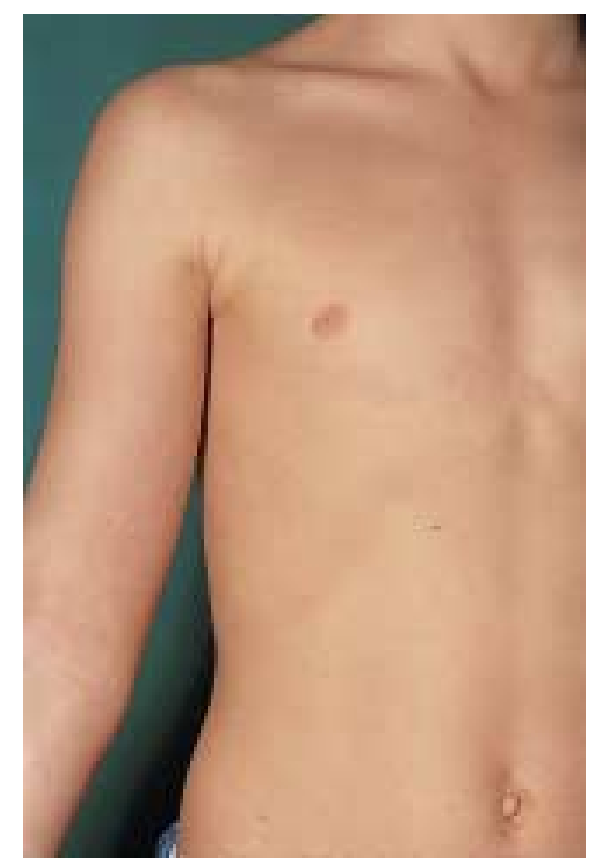

Abb. 5 12 Wochen nach Therapie mit Imiquimod 5\%, Therapiekontrolle nach 3 Monaten.

derzeit nur als $5 \%$ ige Creme (Aldara Creme ${ }^{\circledR}$ ) zur Therapie von genitalen und perianalen Warzen (Condylomata acuminata) zugelassen. Insgesamt deuten die Untersuchungen darauf hin, dass Imiquimod ein weitaus größeres Indikationsspektrum abdecken kann, als bisher bekannt ist.

Die Frage der optimalen Dosierung und Behandlungsdauer für Mollusca contagiosa bei Kindern ist in einer größeren Studie zu klären (Tab. 3, Abb.1-5).

\section{Literatur}

${ }^{1}$ Ahmed I, Berth-Jones J. Imiquimod, a novel treatment for lentigo maligna. Br J Dermatol 2000; 143: 843 - 845

${ }^{2}$ Arevalo I, Ward B, Miller R, Meng TC, Najar E, Alvarez E, Matlashewski G, Llanos-Cuentas A. Successful treatment of drug-resistant cutaneous leishmaniasis in humans by use of imiquimod, an immunmodulator. Clin Infect Dis 2001; 33: 1847-1851 
${ }^{3}$ Bettloch I, Pinazo I, Mestre F, Altes J, Vilialonga C. Molluscum contagiosum in human immunodeficiency virus infection: response to zidovudine. Int J Dermatol 1989; 28: 351 - 352

${ }^{4}$ Beutner KR, Tyring SK, Trofatter KF Jr, Douglas JM Jr, Spruance S, Owens ML, Fox TL, Hougham AJ, Schmitt KA. Imiquimod, a patientapplied immune response modifier for treatment of external genital warts. Antimicrob Agents Chemother 1998; 42: 789-794

${ }^{5}$ Beutner KR, Geisse JK, Heiman D, Fox TL, Ginkel A, Owens ML. Therapeutic response of basal cell carcinoma to the immune response modifier imiquimod 5\% cream. J Am Acad Dermatol 1999; 41: 1002 - 1007

${ }^{6}$ Bornstein J, Pascal B, Zarfati D, Goldshmid N, Abramovici H. Recombinant human interferon-beta for condylomata acuminata: a randomized, double-blind, placebo-controlled study of intralesional therapy. Int J STD AIDS 1997; 8: 614-621

${ }^{7}$ Dahl MV. Imiquimod: An immune response modifier. J Am Acad Dermatol 2000; 43: 1 -5

${ }^{8}$ Diaz Arrastia C, Arany I, Robazeti SC, Dinh TV, Gatalica Z, Tyring SK, Hanningan E. Clinical and molecular responses in high grade intraepithelial neoplasia treated with topical imiquimod 5\%. Clin Cancer Res 2001; 7: 3031-3033

${ }^{9}$ Edwards L, Ferenczy A, Eron L, Baker D, Owens ML, Fox TL, Hougham AJ, Schmitt KA. Self-administered topical $5 \%$ imiquimod cream for external anogenital warts. HPV Study Group Arch Dermatol 1998; 134: $25-30$

${ }^{10}$ Edwards L. Imiquimod in clinical practice. J Am Acad Dermatol 2000; 43: $12-17$

${ }^{11}$ Gilbert J, Drehs MM, Weinberg JM. Topical imiquimod for aciclovirunresponsive herpes-simplex virus 2 infection. Arch Dermatol 2001; 137: $1015-1017$

${ }^{12}$ Hammes S, Greve B, Raulin C. Mollusca contagiosa. Hautarzt 2001; 52: $38-42$

${ }^{13}$ Hengge UR, Esser S, Schultewolter T, Stockfleth E, Meyer T, Arndt R, Goos M. Self administered topical imiquimod for the treatment of common warts and mollusca contagiosa. Br J Dermatol 2000; 143: $1026-1041$

${ }^{14}$ Epstein E. Cantharidin therapy for molluscum contagiosum in children. J Am Acad Dermatol 2001; 45: 638

${ }^{15}$ Funt TR, Mehr KA. Cantharidin: a valuable office treatment of molluscum contagiosum. South Med J 1979; 72: 1019

${ }^{16}$ Imbertson LM, Beaurline JM, Couture AM, Gibson SJ, Smith RMA, Miller RL. Cytokine induction in hairless mouse and rat after topical ap- plication of the immune response modifiers, imiquimod and S-28 463. J Invest Dermatol 1998; 110: 734 - 739

${ }^{17}$ Liota E, Smith KJ, Buckley R, Menon P, Skelton H. Imiquimod therapy for molluscum contagiosum. J Cutan Med Surg 2000; 4: 76-82

${ }^{18}$ Marks R, Gebauer K, Shumack S, Amies M, Bryden J, Fox TL, Owens ML. Imiquimod $5 \%$ cream in the treatment of superficial basal cell carcinoma: results of a multicenter 6-week dose-response trial. J Am Acad Dermatol 2001; 44: 807-813

${ }^{19}$ Meadows KP, Tyring SK, Pavia AT, Rallis TM. Resolution of recalcitrant molluscum contagiosum virus lesions in human immunodeficiency virus-infected patients treated with cidofovir. Arch Dermatol 1997; 133: $987-990$

${ }^{20}$ Mackenzie-Wood A, Kossard S, de Launey J, Wilkinson B, Owens ML. Imiquimod $5 \%$ in the treatment of Bowen's disease. J Am Acad Dermatol $2001 ; 44: 462-470$

${ }^{21}$ Niizeki K, Hashimoto K. Treatment of Mollusca contagiosum with Silver Nitrate Paste. Pediatric Dermatol 1999; 16: 359-397

22 Oster-Schmidt C. Imiquimod: a new possibility for treatment-resistant verrucae planae. Arch Dermatol 2001; 137: 666 - 667

${ }^{23}$ Romiti R, Ribeiro AP, Romiti N. Evaluation of the effectiveness of 5\% potassium hydroxide for the treatment of molluscum contagiosum. Pediatr Dermatol 2000; 17: 495

${ }^{24}$ Sauder DN. Immunomodulatory and pharmacologic properties of imiquimod. J Am Acad Dermatol 2000; 43: 6-11

${ }^{25}$ Smith KJ, Liota E, Yeager J, Menon P. Treatment of molluscum contagiosum with topical imiquimod (abstract 352). Presented at the Annual Meeting of the American Academy of Dermatology, New Orleans, LA, March 19-24, 1999.

${ }^{26}$ Stockfleth E, Meyer T, Benninghoff B, Christophers E. Successful treatment of actinic keratosis with imiquimod cream 5\%: a report of six cases. Br J Dermatol 2001; 144: 1050-1053

${ }^{27}$ Tyring SK, Arany I, Stanley MA, Tomai TA, Miller RL, Smith MH et al. A randomised, controlled, molecular study of condylomata acuminata clearance during treatment with imiquimod. J Infect Dis 1998; 178: $551-555$

${ }^{28}$ Tyring S. Imiquimod applied topically: A novel immune response modifier. Skin Therapy 2001; 6: 1 -4

${ }^{29}$ Zabawaski EJ Jr, Cockerell CJ. Topical cidofovir for molluscum contagiosum in children. Pediatr Dermatol 1999; 16: 414-415 